\title{
Non-C282Y familial iron overload: evidence for locus heterogeneity in haemochromatosis
}

\author{
S Pinson, J Yaouanq, A M Jouanolle, B Turlin, H Plauchu
}

\begin{abstract}
Haemochromatosis (HC) is an autosomal recessive disease with progressive iron overload leading to midlife onset of clinical complications. The causal gene (HFE) maps to 6p, in close linkage with the HLA class I genes. An HFE candidate gene recently identified has two missense mutations (C282Y and H63D) associated with the disease. Here we document the phenotypic and genetic analysis of a nuclear family comprising two sibs with symptomatic and massive iron overload before the age of 25 . The disease seemed to be recessively transmitted and fitted the agreed criteria for haemochromatosis, but was neither associated with the $C 282 Y$ and H63D mutations nor linked with HLA markers. Our data strongly support locus heterogeneity in haemochromatosis by showing evidence that the gene responsible for juvenile haemochromatosis (JH) does not map to $6 \mathrm{p}$. In the absence of clear cut phenotypic distinction from typical haemochromatosis, patients below 30 years of age and $\mathrm{C} 282 \mathrm{Y}$ negative should be considered as putative juvenile cases. This has practical implications in genetic counselling and family management. (F Med Genet 1998;35:954-956)
\end{abstract}

Service de Génétique Clinique, Hôpital de l'Hôtel Dieu, Lyon 69 288, France

$S$ Pinson

H Plauchu

Service

d'Epidémiologie et

Hygiène Hospitalière, Hôpital Pontchaillou, CHRU, 2 Rue Henri Le Guilloux, 35033

Rennes, France

J Yaouanq

Service de Génétique Moléculaire et

Hormonologie, Pontchaillou, Rennes 35033, France

A $M$ Jouanolle

Laboratoire

d'Anatomie et Cytologie

Pathologiques, Pontchaillou, CHRU, Rennes 35033, France B Turlin

Correspondence to: Dr Yaouanq.

Received 14 November 1997 Revised version accepted for publication 3 April 1998

(1) a cysteine to tyrosine substitution at position 282 of the final protein (C282Y), and
(2) a histidine to aspartic acid substitution a position $63(\mathrm{H} 63 \mathrm{D})$. The $\mathrm{C} 282 \mathrm{Y}$ mutation is strongly associated with the disease. The $\mathrm{C} 282 \mathrm{Y}$ homozygous genotype is observed in more than $82 \%$ of white patients from the USA, ${ }^{45}$ Australia, ${ }^{6}$ and Brittany. ${ }^{7}$ The H63Dmutation accounts for a further $5-10 \%$ o $\overline{\hat{\epsilon}}$ patients, who are generally $\mathrm{C} 282 \mathrm{Y} / \mathrm{H} 63 \mathrm{P}$ compound heterozygotes.

Here we document a family comprising two sibs clinically affected with massive iron overload before the age of 25 . The proband (II.1) was a 20 year old man who complained of chronic fatigue and presented with generalisecf skin pigmentation. He had no hepatomegaly or sign of liver failure, no clinical or radiologica arthropathy, and no endocrine or heart failure On biochemical evaluation he had markedly raised serum iron indices and serum amino@ transferase levels (table 1), which were noto alcohol related (no alcohol consumption, nor mal $\gamma$-glutamyl transferase). His body mass index (BMI) was $17.7 \mathrm{~kg} / \mathrm{m}^{2}$ and his glucose metabolism was normal with blood glucose lev els in the fasting state and two hours after 75 g oral glucose loading at 4.9 and $5.7 \mathrm{mmol} / \mathrm{l}$ respectively. Serological markers for hepatitis $A$ $\mathrm{B}$, and $\mathrm{C}$ were negative. Other liver tests were normal, as were haematological parameters (table 1). Liver biopsy showed grade 3-4 hepaच tosiderosis without sideronecrosis but compli cated by extensive fibrosis. Neither hepatocytiç degenerative changes, such as Mallory bodies nor steatosis were found. Iron was predomi nantly deposited in parenchymal cells with decreasing gradient from the periportal to the centrolobular zone. The hepatic iron index (HII=15) was in the range of $\mathrm{HC}^{8}$ as were the tissular iron score (TIS) of 40 and the TIS/age ratio of 2 (normal range 0 to 0.14 ). ${ }^{9}$ The diag $N$ nosis of HC was based upon (1) the above quantitative criteria, (2) the characteristion pattern of hepatic iron distribution, and (3) the absence of any known cause that might confer similar histological features, such as chronic oral intake of iron or ascorbic acid and ineffecs tive erythropoiesis. The patient was treated byo weekly phlebotomies until his serum irone parameters dropped to the low normal limin (serum iron $15 \mathrm{~mol} / \mathrm{l}$, transferrin saturation $\vec{b}$ $20 \%$, ferritin $59 \mu \mathrm{g} / \mathrm{l}$ ), reflecting iron depletion which was achieved after removal of 13 litres of blood (that is, $6.5 \mathrm{~g}$ of iron).

Family testing showed significantly raise of serum iron indices (table 1 ) in the proband's 23 ? year old sister (II.2), whose iron status had never been investigated despite the presence of chronic fatigue and oligomenorrhoea of 8 years' duration. She had menarche at the age of 
Table 1 Iron status, biochemical parameters, and HLA haplotypes of the two affected sibs (II. 1 and II.2) and their parents (I.1 and I.2)

\begin{tabular}{lllll}
\hline & $I .1$ & $I .2$ & $I I .1$ & $I I .2$ \\
\hline Sex & $\mathrm{M}$ & $\mathrm{F}$ & $\mathrm{M}$ & $\mathrm{F}$ \\
Age at first testing (y) & 53 & 49 & 20 & 23 \\
Serum iron $(\mu \mathrm{mol} / \mathrm{l})$ & 11.1 & 21.4 & 57.5 & 50 \\
Transferrin saturation (\%) & - & 33 & 100 & 95 \\
Serum ferritin $(\mu \mathrm{g} / \mathrm{l})$ & 87 & 24 & 1967 & 2575 \\
AST-ALT (UI/) & - & - & $62-115^{\star}$ & $34-30 \dagger$ \\
Haemoglobin (g/dl) & 15.3 & 12.3 & 14.2 & 13.6 \\
Mean corpuscular volume (fl) & 90 & 91 & 92 & 86 \\
Hepatic iron concentration $(\mu \mathrm{mol} / \mathrm{g})$ & - & - & 299 & Not done \\
Total body iron stores $(\mathrm{g})$ & - & - & 6.5 & $>4$ \\
HLA-A, B haplotypes & A2,B18 & A24,B51 & A2,B18 & A11,B55 \\
& A11,B55 & A68,B53 & A68,B53 & A24,B51 \\
\hline
\end{tabular}

Normal values : serum iron $<25 \mu \mathrm{mol} /$, transferrin saturation $<40 \%$, serum ferritin $<300 \mu \mathrm{g} / 1$ hepatic iron concentration $<36 \mu \mathrm{mol} / \mathrm{g}$ dry weight, serum aspartate transaminase (AST) $<35^{\star}$ and $<22 \dagger$, serum alanine transaminase (ALT) $<40^{\star}$ and $<25 \dagger$.

15 , with subsequent irregularly spaced menses occurring at 40 to 120 day intervals. At the time of study, she had never used oral contraceptives or been pregnant, had no history of oral or parenteral iron intake, and was not overweight $\left(B M I=18.1 \mathrm{~kg} / \mathrm{m}^{2}\right)$. Her haematological parameters were normal (table 1), as were inflammatory markers. Physical examination was unremarkable and she refused any endocrine investigation or liver biopsy. However, she underwent phlebotomy therapy and was still not iron depleted (serum ferritin $518 \mu \mathrm{g} / \mathrm{l}$ ) after removal of $4 \mathrm{~g}$ of iron by weekly venesections. Unlike their offspring, the parents (I.1 and I.2) had a normal iron status (table 1) in the absence of any cause which may have delayed the accumulation of iron (no dietary idiosyncrasy, no blood donation, no pathological blood loss or malabsorption). They traced their origin to central France and had no common ancestor identified in their six generation pedigree.

Genetic analysis of the family showed that both the proband and his sister lacked the C282Y and H63D mutations. Furthermore, they did not share any parental HLA haplotype (table 1). These unexpected findings warrant discussion with respect to the possible aetiology of the disease segregating within the family. Careful re-examination of phenotypic data confirmed the absence of any known cause of secondary iron overload. Iron associated disorders, including hepatitis $\mathrm{C}$ and acaeruloplasminaemia, were easily excluded. Steatohepatitis was also ruled out, since neither of the two sibs had the usually associated features (no overweight, no abnormality of glucose and lipid metabolism, no arterial hypertension) and the proband had no liver fat deposition or Mallory bodies. Furthermore, none of the usual causes of increased iron uptake except for haemochromatosis could explain the distribution of hepatic iron in the proband, whose disease fitted the accepted phenotypic criteria for $\mathrm{HC}$ (HII $>1.9$, TIS/age $>0.15$, and total of body iron excess $>5 \mathrm{~g}) .^{8-10}$ The significant increase of body iron stores $(>4$ g) in the clinically affected sister further supported the diagnosis of HC. This was not incompatible with the observation that both sibs were negative for $\mathrm{C} 282 \mathrm{Y}$ and H63D. Indeed, a significant proportion of well defined haemochromatosis patients lack C282Y, some of whom are also negative for H63D. Such "negative" cases account for 7-9\% of patients in the USA ${ }^{45}$ and southern France, ${ }^{11}$ and up to $21 \%$ in Italy. ${ }^{12}$ Since no additional mutation has been found except in one case, ${ }^{13}$ other gene(s) potentially relevant for haemochromatosis are expected to be discovered within the extensive linkage disequilibrium zone telomeric to HLA-A. ${ }^{414}$ However, the present pedigree further complicates the problem since the affected sibs were totally discordant for HLA-A and -B alleles.

Despite recessive inheritance and HLA linkage, not all haemochromatosis sib pairs are identical by descent for two HLA parental haplotypes. ${ }^{15}$ This is usually taken to indicate more than two HFE alleles in the parental gene pool, a situation which frequently arises in populations with a high gene frequency. Crossover events between HFE and HLA-A, although very rare, could account for some additional cases. All these possibilities were ruled out in our family. First, HLA typing of the two parents allowed the unambiguous definition of the four HLA parental haplotypes. Secondly, homozygosity for HC of the father was unlikely, because within affected families this genotype has an estimated penetrance of $100 \%$ in males over $40 .^{16}$ Then, by postulating heterozygosity for $\mathrm{HC}$ in both parents, one would suppose that two independent crossover events separating HFE and HLA-A have occurred (one in each meiosis, or two in only one meiosis), which is incompatible with the extreme rarity of recombination in the genomic region considered. ${ }^{4}{ }^{17}$ Alternatively, if the father is a heterozygote and the mother a nonexpressing homozygote, a crossover event would have occurred in the paternal meiosis leading to II.1 or II.2. This possibility is weakened by the fact that the HLA-A11 paternal haplotype belongs to a category of "stable" haplotypes, whose high degree of conservation is probably the result of a mechanism of recombination suppression. ${ }^{18}$ Finally, rejection of the classical (HLA linked) form of haemochromatosis is by far the most likely explanation. The early onset of significant iron accumulation in the two sibs, and dysmenorrhoea in the sister, are consistent with juvenile haemochromatosis $(\mathrm{JH})$, despite the absence of cardiac dysfunction and the degree of iron overload which might appear lower than in some of the rare reported cases. ${ }^{319}$ On the whole, the present pedigree data indicate that the disease might be inherited as an autosomal recessive trait but does not segregate with markers on $6 \mathrm{p}$. This provides evidence that the molecular defect(s) responsible for JH must be searched for outside the $6 \mathrm{p}$ chromosomal region, as recently stated by the Italian group. ${ }^{19}$ Collaborative studies should indicate whether our two cases and those born from consanguineous Italian parents ${ }^{19}$ are genetically different.

The clear demonstration that at least one gene unlinked to $6 \mathrm{p}$ causes a haemochromatosis phenotype in the young has important practical implications in genetic counselling. Obviously, screening for the HFE mutations and family gene tracking with $6 \mathrm{p}$ markers are 
inaccurate for predicting genotypes at risk for $\mathrm{JH}$. On the other hand, the frequency of the disease may have been underestimated, since it may be difficult to distinguish juvenile and typical haemochromatosis on the sole basis of a different rate of iron accumulation. ${ }^{3}$ In practice, the risk for having unrecognised $\mathrm{JH}$ is mainly restricted to the group of patients under 30 years who are negative for $\mathrm{C} 282 \mathrm{Y}$, so that any proband in this group should be considered as a putative juvenile case. Then, until the identification of the underlying molecular defect(s) allows unambiguous distinction from the HLA linked form, his/her first degree relatives should be carefully examined, especially unaffected sibs whose iron status should be periodically tested regardless of the number of $6 \mathrm{p}$ parental haplotypes they share with the proband. Together with the recent suggestion that certain $\mathrm{C} 282 \mathrm{Y}$ homozygotes may remain unaffected, ${ }^{20}$ the increasing evidence of locus heterogeneity in primary iron loading disorders will have a critical impact on future diagnosis and screening strategies, especially in ethnically heterogeneous populations.

We are grateful to the members of the family for their cooperation. We thank Professor J C Chuat for carefully reading the manuscript.

1 Simon M. Disorders of iron metabolism and related disorders. In: Emery AE, Rimoin DL, eds. Principles and practice of medical genetics. 2nd ed. Edinburgh: Churchill Livingstone, 1990:1783-96.

2 Lamon JM, Marynick SP, Rosenblatt R, Donnelly S. Idiopathic hemochromatosis in a young female. A case study and review of the syndrome in young people. Gastroenterology 1979;76:178-83.

3 Kaltwasser JP, Schalk KP, Werner A. Juvenile hemochromaKaltwasser JP, Schalk KP, Werner A. Juvenile hemochroma-
tosis. In: Weintraub LR, Edwards CQ, Krikker M, eds. Hemochromatosis. Proceedings of the first international conference. Ann N Y Acad Sci 1988;526:339-41.

4 Feder JN, Gnirke A, Thomas W, et al. A novel MHC class I like gene is mutated in patients with hereditary haemochromatosis. Nat Genet 1996;13:399-408.
5 Beutler E, Gelbart T, West C, et al. Mutation analysis in hereditary hemochromatosis. Blood Cells Mol Dis 1996;22: 187-94.

6 Jazwinska EC, Cullen LM, Busfield F, et al. Haemochromac tosis and HLA-H. Nat Genet 1996;14:249-51.

7 Jouanolle AM, Gandon G, Jezequel P, et al. Haemochroma tosis and HLA-H. Nat Genet 1996;14:251-2.

8 Bassett ML, Halliday JW, Powell LW. Value of hepatic iron measurements in early hemochromatosis and determina $\frac{\mathbb{D}}{7}$ tion of the critical iron level associated with fibrosis. Hepaed tology 1986;6:24-9.

9 Deugnier YM, Turlin B, Powell LW, et al. Differentiation between heterozygotes and homozygotes in genetic hemos chromatosis by means of a histological hepatic iron index: study of 192 cases. Hepatology 1993;17:30-4.

10 Deugnier YM, Loreal O, Turlin B, et al. Liver pathology in genetic hemochromatosis: a review of 135 homozygous? cases and their bioclinical correlations. GastroenterologxD 1992;102:2050-9.

11 Borot N, Roth MP, Malfroy L, et al. Mutations in the MHCO class I-like candidate gene for hemochromatosis in French patients. Immunogenetics 1997;45:320-4.

12 Carella M, D'Ambrosio L, Totaro A, et al. Mutation analy: sis of the HLA-H gene in Italian hemochromatosispatients. Am f Hum Genet 1997;60:828-32.

13 Pointon J, Sherman JD, Merryweather-Clarke AT, Robson $\mathrm{KJH}$. A single nucleotide deletion in the putative haemochromatosis gene in a patient who is negative for both the C282Y and H63D mutations. Proceedings of the international symposium: Iron in biology and medicine, $\mathrm{St}$ Malo, France, 1997:268.

14 Tay GK, Leelayuwat C, Chorney MJ, et al. The MHC conzio tains multiple genes potentially relevant to hemochromatos sis. Immunogenetics 1997;45:336-40.

15 Powell LW, Bassett ML, Axelsen E, Ferluga J, Halliday JW윽 Is all genetic (hereditary) hemochromatosis HLA= associated? In: Weintraub LR, Edwards $\mathrm{CQ}$, Krikker $\mathrm{M}_{\text {, }}$ eds. Hemochromatosis. Proceedings of the first inter national conference. Ann N Y Acad Sci 1988;526:23-33.

16 Borecki IB, Rao DC, Yaouanq J, Lalouel JM. Serum ferritin as a marker of affection for genetic hemochromatosis. Hum Hered 1990;40:159-66.

17 Ajioka RS, Jorde LB, Gruen JR, et al. Haplotype analysis of haemochromatosis: evaluation of different linkage disequilibrium approaches and evolution of disease chro€ mosomes. Am f Hum Genet 1997;60:1439-47.

18 Gandon G, Jouanolle AM, Chauvel B, et al. Linkage disequilibrium and extended haplotypes in the HLA-A to D6S105 region: implications for mapping the hemochro matosis gene (HFE). Hum Genet 1996;97:103-13.

19 Camaschella C, Roetto A, Cicilano $M$, et al. Juvenile ang adult hemochromatosis are distinct genetic disorders. Eur Hum Genet. 1997;5:371-5.

20 Rhodes DA, Raha-Chowdhury R, Cox TM, Trowsdale Homozygosity for the predominant Cys282Tyr mutation and absence of disease expression in hereditary haemo 3 chromatosis. F Med Genet 1997;34:761-4. 